\title{
GRAVITY AND POSITIONAL HOMEOSTASIS OF THE CELL
}

\author{
George W. Nace \\ Division of Biological Sciences and Center for Human Growth and \\ Development, The University of Michigan, Ann Arbor, MI, U.S.A.
}

\section{ABSTRACT}

Normally bilateralization takes place in the presence of the Earth's gravity which produces torque, shear, tension and compression acting upon the naked aggregates of cytoplasm in the zygote which is only stabilized by a weak cytoskeleton. In an initial examination of the effects of these quantities on development, an expression is derived to describe the tendency of torque to rotate the egg and reorganize its constituents. This expression yields the net torque resulting from buoyancy and gravity acting upon a dumbbell shaped cell with heavy and light masses at either end and "floating" in a medium. Using crude values for the variables, torques of $2.5 \times 10^{-13}$ to $8.5 \times 10^{-1}$ dyne-cm are found to act upon cells ranging from $6.4 \mathrm{~mm}$ to $31 \mathrm{~mm}$ (chicken egg). By way of camparison six microtubules can exert a torque of $5 \times 10^{-9}$ dyne-cm. (1) Gravity imparts torque to cells; (2) torque is reduced to zero as gravity approaches zero; and (3) torque is sensitive to cell size and particulate distribution. Cells must expend energy to maintain positional homeostasis against gravity. Although not previously recognized, skylab 3 results support this hypothesis: tissue cultures used $58 \%$ more glucose on Earth than in space. The implications for developmental biology, physiology, genetics, and evolution are considered. At the cellular and tissue level the concept of "gravity receptors" may be unnecessary.

\section{INTRODUCTION}

Contemplating the relationships among the gravitational force, the orientation of the egg relative to the direction of gravity, and the sperm entry point as factors in the establishment of the bilaterality of the frog zygote [1-4], I was led to a consideration of the forces which might be involved. This resulted in the realization (1) that the force exerted upon cells by gravity tends to induce cellular deformations or changes in orientation; (2) that the consequences of this action can best be appreciated through a concept of positional homeostasis of the cell; (3) that the level of energy utilization by the cell to maintain positional homeostasis may be the only immediate evidence of its response to gravitational change; and (4) that the energy expended for positional homeostasis represents a costly component of basal metabolism at Earth gravity.

The matters under consideration apply to the action of gravity upon cytoplasmic aggregates of the size present in eggs, and upon many kinds of cells and larger units of biological structure. The effects of the action of gravity on cells the size of bacteria (average size $1.5 \mathrm{\mu m}$ ), or cellular structures such as ribosomes, or molecular constituents of cells drop rapidly toward zero as the size of the particle decreases. This evaluation derives from Pollard [5] who has shown that when small masses are involved (below about $10 \mu \mathrm{m}$ ), the rapid random motion caused by Brownian movement increasingly overcomes the "slow, purposeful motion induced by gravity." However, depending upon their orientation to gravity, structures from about 5 to $10 \mu \mathrm{m}$ and larger tend to be twisted, flattened or stretched.

The literature is replete with evidence that for normal function cells must maintain specific shapes and positions: defined cellular interactions are critical to early embryogenesis; cells in tissues must maintain precise orientations relative to their sources of nutrients, the objects of their 
actions, and the depots for their secretions; cells in culture change their products depending upon the physical relationships established among them [6]. Other examples abound. It is evident, then, that positional homeostasis, or the maintenance of the cell's normal presentation to its environment, is essential to normal function and that the cell or its neighbors must expend energy in maintaining its shape and position to the extent that gravity tends to degrade this presentation.

The energy for positional homeostasis must be expended in the fabrication, maintenance and use of structures which provide motility or which hold the cell in specific relationship to its environment. If a cell exposed to modified gravity (such as in space or on a centrifuge) already has structures and biochemical mechanisms adapted to this purpose, the only evident change in response to the modified gravity may be the level of energy expenditure. If, however, the available mechanisms are not appropriate to the demands of positional homeostasis, either deficient or in excess, feedback control systems, perhaps operating at the genetic level, must certainly be activated to modify the machinery of the cell in order to adapt it to the levels of energy required for positional homeostasis. If such modifications do occur, they should be evident in cells exposed to gravitational change. Such responses would be most pronounced during early development, such as at the time of bilateralization, when mechanisms involved in cell stabilization are poorly elaborated.

This paper presents these concepts, which are of significance for the design of biological experiments in space, and cites suggestive evidence from experiments conducted on skylab 3. Torque is arbitrarily chosen as the quantity to illustrate the action of gravity as its description is straightforward, reasonable values for the parameters needed for its calculation are available, and it plays a particularly significant role in the bilateralization of the egg. It may, however, represent only a small part of the tendency of gravity to deform and displace cells and cell assemblages. Other relevant quantities such as shear, tension, compression and positional translation will be considered in another context.

\section{THE PHYSICS OF GRAVITATIONAL ACTION ON CELIS}

Torque, $L$, the moment of force, is a measure of the tendency of a force to produce rotation about an axis. It is calculated as

$$
I=F d \sin \theta,
$$

where $F$ is force; $d \sin \theta$, the lever arm, is the product of distance, $d$, measured from the center of the mass upon which the force is applied to the axis of rotation, and $\sin \theta$, in which $\theta$ is the angle between $F$ and $d$. In the cgs system the unit of $F$ is the dyne or the force needed "to give acceleration of one centimeter per second per second to one gram of mass"; d is measured in centimeters; so that the unit of $L$ is a dyne-centimeter. Note that the orientation of $d$ with respect to the direction of the gravitational force is of significance as it changes the value of $d \sin \theta$.

Work (in ergs) is Fs, force acting through a distance, s; torque is force acting on a lever arm. Displacement is a prerequisite for work, but not for torque. Work done in rotation is $L \theta$, where $\theta$ is the angle through which the torque acts.

For the sake of simplicity we will assume a symmetrical, elongated cell immersed and free-floating in a fluid medium on Earth where the acceleration of gravity is about $980 \mathrm{~cm} / \mathrm{sec}^{2}$. In an initial approximation, to avoid complications resulting from consideration of the relationship between the cell surface and the medium, we will think of the cell as a dumbbell (Fig. I). This dumbbell is a shell. The balls at each end displace medium and thus produce buoyancy. We will think of the mass of the cell as aistributed in the form of a solid dumbbell within the shell, with one end, $H$, containing dense storage granules as the "solute", the other end, L, lighter than the medium, containing oil vacuoles as the "solute." The volumes of the masses at each end of the solid dumbbell, $V_{\underline{H}}$ and $V_{\underline{L}}$, are concentric with the balls of the shell but need not be equal. Thus we' take $\mathrm{V}_{\mathrm{H}} \leq \mathrm{V}_{B H}>\mathrm{V}_{B L}>\mathrm{V}_{L}$ where $V_{B H}>0$ and $V_{H}$ and $V_{L}$ are both $\geq 0$. We will also consider the ceIl to be rigid, both along its $\overline{a x i s}$ and in its orientation to gravity, for the instant in time to which the calculation applies, recognizing that cells are dynamic 


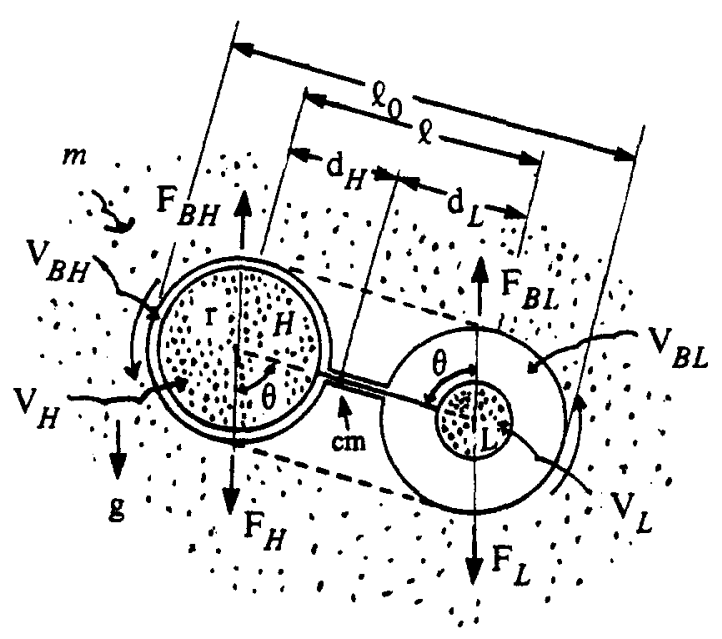

Fig.l The floating cell model used in this study. H and $I$ are the heavy and light masses of the cell. I is the radius of the heavy mass. $l_{0}$ is the length of the cell; $l$, the distance between the centers of the heavy and light masses; $d_{y}$ and $d$, the distances from these centers, respectively, to the center of mass, c.m., of the cell. $V_{H}$ and $V_{L}$ are the volumes of $H$ and $I$ and $V_{B H}$ and $V_{B I}$ are the volumes of the buoyant spheres surrounding $V_{H}$ and $V_{I}, F_{H}$ and $F_{I}$ are the force of gravity and $F_{B H}$ and $F_{B I}$ are the büoyant forces, respectively, acting on $\underline{H}$ and $\bar{I}$. $g$ shows the direction of gravity and $\theta$ is the angle between $F$ and $l$. $m$ is the medium in which the cell is suspended.

and that for longer intervals shape distortion and tumbling would occur. These would necessitate the consideration of additional factors which would not change the conclusions of this treatment.

Such a cell tends to rotate about its center of mass, c.m., so that the sum of the torques is

and from (1)

$$
\begin{gathered}
I_{S}=I_{\underline{H}}-I_{L^{\prime}} \\
I_{S}=F_{\underline{H}} d_{\underline{H}} \sin \theta-F_{\underline{I} \underline{I}} \sin \theta .
\end{gathered}
$$

Force is

$$
F=m a
$$

where $\mathrm{m}$ is mass in grams and $\mathrm{a}$ is linear acceleration in $\mathrm{cm} / \mathrm{sec}^{2}$, and, in this case, is $g$, the acceleration due to gravity.

Mass equals the product of its volume, $V$, and its density, $p(g / c c)$, or

$$
m=V \rho \text {. }
$$

But, the effective mass is influenced by buoyancy, the weight of the fluid displaced, $V_{B} \rho m$, where $\rho m$ is the density of the medium. In fact, for the solutes whosé masses are $\mathrm{m}_{\underline{\underline{H}}}$ and $\mathrm{m}_{\underline{L}}$,

$$
\text { Effective mass of } \underline{\underline{H}}={\underline{m_{H}}}_{\underline{H}}-{\underline{V_{B H}}} \underline{\underline{m}}
$$

and

From (3), (4), (6) and (7) net torque is

$$
\text { Effective mass of } \underline{I}=\mathrm{m}_{\underline{L}}-\mathrm{V}_{\underline{B L}^{\rho} \underline{m}} \text {. }
$$

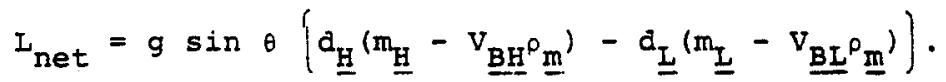


The distance, $\ell$, between the centers of each mass is the sum of $d_{H}$ and $d_{L}$, the distances from the centers of each mass to the axis of rotation. Lengths $\mathrm{d}_{\mathrm{H}}$ and $\mathrm{d}_{\mathrm{L}}$, in turn, are the fractions of $\&$ determined by the proportion of eâch mas $\bar{s}$ to the total mass, and, measuring to the axis of rotation from $\mathrm{m}_{\mathrm{H}}$ for $d_{\underline{H}}$ and from $m_{\underline{L}}$ for $d_{\underline{L}}$, are

$$
\begin{aligned}
& d_{\underline{H}}=\ell m_{\underline{I}} /\left(m_{\underline{H}}+m_{\underline{L}}\right) \\
& d_{\underline{L}}=\ell m_{\underline{H}} /\left(m_{\underline{H}}+m_{\underline{L}}\right) .
\end{aligned}
$$

Substituting (9) and (10) in (8) and rearranging gives

$$
I_{n e t}=g l \sin \theta \quad \rho_{\underline{m}}\left(\frac{\underline{m_{y} V_{B L}-m_{I} V_{B H}}}{m_{\underline{H}}+m_{\underline{L}}}\right) \text {. }
$$

Using (5) to express (11) in terms of $V$ and $o$ gives

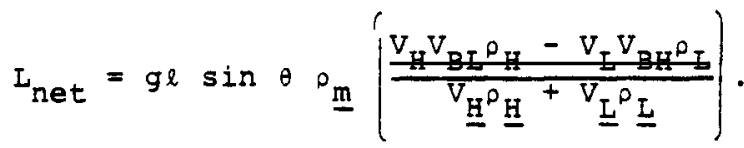

Expressed as radii, r, of the respective volumes, (12) becomes

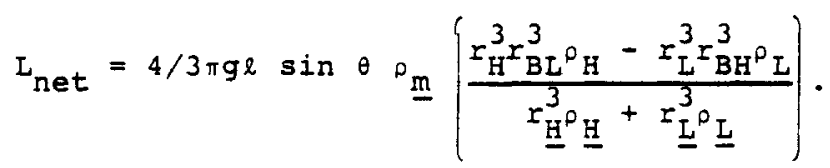

But,

$$
\ell=a \ell_{0} \quad \text { when } 0<a<1
$$

and, from the volume relationships specified above

$$
\begin{aligned}
& r_{\underline{H}}=b r_{B H} \quad \text { when } 0<\mathrm{b} \leq 1, \\
& r_{B L}=c r_{B H} \quad \text { when } 0<c \leq 1, \\
& r_{\underline{B L}}=d r_{\underline{B L}} \text { when } 0<\mathrm{d} \leq 1 .
\end{aligned}
$$

Then, by substitution, (13) becomes

$$
L_{\text {net }}=4 / 3 \pi g a \ell_{0} c^{3} r_{\underline{B H}}^{3} \sin \theta \rho_{\underline{m}}\left[\frac{b^{3} \rho_{H}-d^{3} \rho_{L}}{b^{3} \rho_{\underline{H}}+c^{3} d^{3} \rho_{L}}\right] .
$$

It is evident that in addition to torque, the cell must contend with tension, shear and compression between $\mathrm{mL}$ and $\mathrm{m}_{\mathrm{H}}$ as $\theta$ changes from $0^{\circ}$ to $180^{\circ}$. As arguments concerning these quantities accent the conclusions of this presentation, they are not treated here. We will also disregard Stoke's Law which gives the rate of fall of a small sphere in a viscous fluid under the force of gravity, viscosity which considers internal friction in the system, and surface tension which considers tension in the surface of separation between the cell and the medium. This is possible because, although these parameters influence the displacement and the response time of the cell, they do not change the torque attributable to gravity at the instant under consideration. Brownian movement, another source of force which, although random, influences cells, is, as noted in the introduction, too small a force to significantly influence cells and structures of the size under consideration here. 


\section{ILLUSTRATIVE ROTATIONAI EFFECTS OF GRAVITY ON CELLS}

To give concrete meaning to this model and to illustrate values of torque for hypothetical cells on Earth, we will assume $\mathrm{mH}_{\mathrm{H}}$ and $\mathrm{mL}$ are separated by $3 / 4$ the length, $\ell_{0}$, of the cell $(a=3 / 4)$, are of equal volume and fill the buoyant volumes $(b=c=d=1)$, are spherical with radii $1 / 8$ the length of the cell $\left(r_{\mathrm{BH}}=\ell_{0} / 8\right)$, and are horizontal $\left(\theta=90^{\circ}\right)$ at the instant of measurement. With these assumptions (14) is simplified to

$$
I_{\text {net }}=\pi g \ell_{0}^{4} / 8^{3} \rho_{\underline{m}}\left(\frac{\rho_{H}-\rho_{I}}{\rho_{\underline{H}}+\rho_{\underline{L}}}\right)=6 l_{0}^{4} \rho_{\underline{m}}\left[\frac{\rho_{H}-\rho_{I}}{\rho_{\underline{H}}{ }^{+\rho_{I}}}\right] \text {. }
$$

Let us choose as a hypothetical example a cell $6.4 \mu \mathrm{m}$ long (e.g., the size of a sarcoma cell [7]). This cell is chosen as its size is near the upper imit for the size of bacteria, near the lower limit for the size of animal and plant cells, and near the value, $10 \mu \mathrm{m}$, below which Pollard [5] suggests the action of Brownian movement may overpower the action of gravity. This cell is in peritoneal fluid $\left(\rho_{\underline{m}}=1.012[8]\right)$ with glycogen granules $\left(\rho_{\mathrm{H}}=1.51[9]\right)$ at $\mathrm{m}_{\mathrm{H}}$ and oil vacuoles $\left(\rho \bar{I}=0.918\right.$ [9]) at $\mathrm{m}_{\mathrm{L}}$. We find this cell tends to rotate with a Inet of $2.5^{-} \times 10^{-13}$ dyne-cm. To appreciate the order of magnitude of this torque it is of interest that the 6 microtubules in each axopodium of the heliozoan, Heterophrys imarina, generate a total force of $10^{-5}$ or $10^{-6}$ dynes [10]. Operating on the lever arm of the $6.4 \mu \mathrm{m}$ cell this would yield a torque of $\sim 5 \times 10^{-9}$ dyne-cm; much smaller if the actual lever arm of a microtubule were known.

Examining the influence of the medium by substituting sea water $(0 \mathrm{~m}=1.025$ [11]) for the peritoneal fluid around this same cell, also yields $\overline{2} .5 \times 10-13$ dyne-cm. Substituting a larger cell (e.g. epidermal cell), $30 \mu \mathrm{m}$ in diameter [7] in peritoneal fluid, keeping other factors the same, yields a torque of $1.2 \times 10^{-10}$ dyne-cm.

Using the $30 \mu \mathrm{m}$ cell but attaching its heavy end to a perpendicular substrate, and adjusting the assumptions concerning the axis of rotation by modifying expression (8), yields Inet of $9.4 \times 10^{-11}$ dyne-cm; or attaching it at the light end yields $L_{n e t}$ of $29 \times 10^{-11}$ dyne-cm.

We turn now to frog eggs as representatives of large cells and use expression (14). Take for Rana pipiens an egg diameter, $\ell_{0}$, of $1700 \mu \mathrm{m}, \mathrm{a} \rho_{\mathrm{H}}$ of 1.3 and a relative $V_{H}$ of $\frac{1}{3}$ for the vegetal region and $a D_{L}$ of 0.9 and a rêlative $V_{I}$ of 1 for the animal region. These $\rho$ and $V$ values are based on sucrose gradient centrifugation studies of $R$. pipiens eggs $[12,13]$. $\rho_{m}$ for periviteliine fluid is chosen as 1.007 based on the specific gravity of amniotic and duodenal fluid [8]. The constants $a, b, c, d$, and $\sin \theta$ are, respectively, 0.5 , $1,0.693,1$ and 1 . $I_{B H}$ is 0.05 . Using these estimates, the $I_{n e t}$ for $R$. pipiens eggs is $3.7 \times 10^{-3}$ dyne-cm or $1.5 \times 10^{10}$ times the torque tending to revolve the $6.4 \mathrm{~km}$ sarcoma cell in peritoneal fluid. Assuming for Xenopus laevis an egg diameter of $1450 \mu \mathrm{m}$ [14] and the other values as for $\bar{R}$. pipiens, inet is $2.0 \times 10^{-3}$ dynes-cm.

At a greater extreme we examine the oocyte, yolk, of the chicken, Gallus gallus [15]. Its polar diameter, $\ell_{0}$, is $31 \mathrm{~mm}$ with the blatodisc anc nucleus of Pander occupying a volume with a radius, rI, of $3 \mathrm{~mm}$ and a $\rho_{1}$ of 1.027 . The remainder of the yolk has a radius, $r_{H}$, of $12.5 \mathrm{~mm}$ and a $\rho_{H}$ of 1.032 . This yolk floats in an albumin medium with a $\rho_{m}$ of 1.04 . The constants $a, b$, $c, d$ and $\sin \theta$ of expression (14) are, respectively, 0.5, 1, 0.24, 1 and 1 . These values generate an Inet of $8.5 \times 10^{-1}$ dyne-cm for the chicken egg.

The same calculations may be attempted on a human egg, diameter $89 \mathrm{\mu m} \mathrm{[7],} \mathrm{if}$ one assumes a polarized heterogeneity which, aside from the eccentric location of the nucleus, has not been seriously investigated. Choosing conservative values of $\rho_{H}=1.1, \rho_{L}=0.95, \rho_{\mathrm{m}}=1.007$, relative $V_{H}=2$ and relative $V_{L}=1$ (compare $\bar{w}_{i t h}$ the vălues for $\underline{R}^{-}$pipiens chosen above) yields a $I_{n e t}$ of $1.5 \times 10^{-8}$ dyne-cm.

Several things are evident from these examples, even with the imperfections in the values chosen for the various parameters: (1) gravity imparts torque to cells, (2) since this torque is proportional to $\mathrm{g}$, it is reduced to zero as gravitational acceleration is reduced to zero, and (3) the torque is highly sensitive to the size of the cell and the distribution of its subcellular particulates. 


\section{POSITIONAL HOMEOSTASIS AND THE IRRELEVANCE OF "GRAVITY} RECEPTORS" AT THE CELLULAR LEVEI

As noted in the introduction, the presentation of a cell to its environment, including neighboring cells, is critical to the normal development and function of all but the most primitive cells and certain specialized cells such as erythrocytes. It may be assumed that to assure appropriate presentation; free cells and cells in organized assemblages have evolved mechanisms working against the force of gravity which tends to shift them away from necessary relationships. The mechanisms suggested by conclusion (3), above, are for the cell to limit its size (note that torque bears a fourth power relationship to cell length) and to minimize the polarization of distribution of its high and low density particulates. As functional considerations often dictate cell length and polarization of particulates, cilia, flagella, pseudopodia and other cellular projections can serve to stabilize free cells, while ground substances, cellular interdigitations, other intercellular structures and packing within an assembly can serve this purpose for multicellular systems. In the case of both free cells and cells in multicellular systems, intracellular structures such as microfilaments and microtubules would also be involved as energy transducers.

Hypothesis 1: Energy, to the extent needed to counteract the action of gravitational force, must be expended by the cell to maintain its position dynamically or by the cell or its neighbors to create and maintain the structures involved in holding it in position.

In the absence of gravity, as in space, this requirement for energy expenditure is reduced to zero.

This hypothesis does not imply or require any changes in morphology or biochemistry in response to gravity, only that greater or lesser energy be expended to maintain the homeostatic state; in this case the homeostasis of position.

Note also that this hypothesis does not exclude the possibility of structural or biochemical change. In the introduction it was pointed out that if cells exposed to gravitational change possessed the structures and biochemical mechanisms needed for positional homeostasis the only response of the cell to the gravitational change (either increase or decrease) would be the changed level of energy expenditure. There would be no morphological evidence of this changed energy expenditure. Also, that if the gravitational change (increase, changed orientation) required stabilization beyond the capacity of the available machinery, feedback control systems, perhaps acting at the genetic level, would be activated to produce the required machinery and this would result in morphological change. Depending upon the demands of the system, this morphological change might only involve changes in the numbers of microfilaments and microtubules. Beyond this, gravitational change (decrease, clinostat) which reduced the energy requirements for positional homeostasis might, through feedback control, inhibit the maintenance and replacement of structures and biochemical mechanisms involved in positional homeostasis. The feedback control response to increased demands (microgravity to $1 \mathrm{~g} ; ~ 1 \mathrm{~g}$ to centrifuge or repositioning) on positional homeostasis may be expected to be quite rapid while the response to decreased demands (centrifuge to $1 \mathrm{~g}$, I $g$ to microgravity or clinostat) would not be driven by necessity a i might only become evident after an extended period. On the other hand, positional homeostasis may be only one of the functions of the mechanisms under consideration. In that case feedback control emanating from these other functions could maintain and replace these mechanisms independent of the demand for positional homeostasis and, aside from changes in enerqy expenditure, there would be no evidence of adaptation regardless of the gravitational exposure. These concepts are amenable to experimental evaluation.

By way of reiteration, all cells from about $10 \mathrm{\mu m}$ or larger [5] are significantly subjected to the force of gravity and energy must be expended dynamically or in the fabrication and maintenance of structures to counteract this influence; otherwise the presentation of the cell to its environment will be modified. Indeed, since cells are increasingly subjected to displacement by Brownian movement as size decreases below about $10 \mu \mathrm{m}$, and since they are subjected to an ever greater tendency to distortion or displacement by gravitational force as size increases (fourth power function of cell length in the case of torque) the combined effects of Brownian movement and gravity may be important factors, along with others such as diffusion distance and surface/ volume relationships, in determining that so many cells fall in the size 
range of 10 to $30 \mu \mathrm{m}$. Special gravity receptors, transducers and communications systems are not needed as the cell itself is, as suggested by pollard [5], both receptor and responder. The action of positional homeostasis integrated over all the cells in a tissue or structure may suffice to produce a tissue or organ response if the gravitational force is changed. The search For the "gravity receptor" may be an unnecessary exercise, although the existence of specialized gravity receptors for complex integrated systems is not excluded.

IMPLICATIONS FOR STUDIES OF WEIGHTLESSNESS ON CEIIS AND EXPERIMENTAL SUPPORT OF THE HYPOTHESIS

Two circumstances have inhibited the space program from undertaking studies on the influence of gravity at the cellular level. One of these has been the work of Pollard [5] which showed that Brownian movement dominated gravitational forces at the level of subcellular particulates and bacteria. The other has been the work by Montgomery and his colleagues [16] that did not recognize differences between tissue cultures flown in Skylab 3 and maintained on Earth.

One must agree that "weightlessness should not be studied in bacterial cells" [5] for which there is no evidence that positional homeostasis is of importance, but also one must not forget Pollard's comment that for larger cells "the situation is altogether different." For larger cells, or cells for which positional homeostasis is of importance, whether in the free-floating state, attached, or in assemblages, studies under conditions of weightlessness or hypergravity may be revealing.

The work by Montgomery et al [16] must be reinterpreted in the light of the considerations presented in this paper. They conducted a wide variety of morphological and biochemical analyses on human cells flown in Skylab 3 for from 1 to 59 days and on control cultures maintained on Earth. They concluded:

"There are no differences between the flight and control cell cultures.

"Minor unexplained differences have been found in biochemical constituents of the used flight and control media. Our present opinion is that these changes are of no significance.

"Within the limits of the experimental design, it was found that a zerogravity environment produced no detectable effects on Wistar-38 human embryonic lung cells in tissue culture."

Examining their text for the unexplained differences found in the media one finds: "There is an unexplained difference in glucose concentration in the used control culture medium $(75 \mathrm{mg}$ percent) and the flight culture medium $(93 \mathrm{mg}$ percent)." Fresh medium contained giucose at "124.0 mg percent." Thus the control cells used "49 mg percent" while the flight celis used "31 mg percent." No other datum in this study was directly related to energy expenditure on the part of the cells in these cultures.

There are some difficulties in evaluating this skylab 3 result on glucose in the culture media as flight opportunities have not been available for confirmatory experiments. It is tempting, therefore, to write it off as an uncontrolled experimental situation. However, this begs the fact that they [16] report remarkably consistent data for 27 constituents in fresh medium and in replicate flight and control media, especially since greater reliability can be expected of analyses for glucose than for many of the other constituents. The fact that of ali the results presented in tabular form, these authors addressed only the glucose results in the text, and, using the expression "our present opinion", commented upon them again in the conclusions, suggests that their denial of significance for the results arose not from lack of confidence in the analyses but from the lack of a conceptual basis for recognizing significance.

Montgomery et al [16] also reported that no differences between flight and control cultures were found for cell attachment, confluency, migration, layering, elongation, alignment, membrane movement, and several other structural parameters. All these activities require energy expenditure in a gravitational field. They also reported that the entoplasm was rich in microtubules and microfilaments although these were not quantitated. 
Accepting their data for the reasons stated, it would seem that contrary to their conclusion, Montgomery et al. [16] presented evidence for an effect of a zero-gravity environment on these cells. Their data suggest that the Earth-bound cells used 58 s more glucose, $i . e .100(49-31) / 31$, and thus an equivalenty greater amount of energy, than did the flown cells. Their results $f$ it the hypothesis presented above remarkably well: Cells relieved of the need to expend energy in maintaining positional homeostasis seemed to use less energy and, as predicted, this decreased energy expenditure was not, during this 59 day period, accompanied by changes in structure or biochemical mechanisms. The surprise is that the energy needed for positional homeostasis was as great as their data seemed to imply. Undoubtedly torque was not the only effect of gravity responsible for this energy expenditure. Other relevant quantities are noted in the Introduction. Perhaps it should be expected, however, when one considers the force of contraction generated by the integration over an entire muscle mass of the small torques produced by each bridge between muscle filaments $\left(2.6 \times 10^{-13}\right.$ dyne-cm calculated from data presented in the literature $[17,18])$, or by the fact that the body must counteract the torque produced by gravity when it acts on any structural component of the body (e.g. $3.6 \times 10^{7}$ dyne-cm on the human forearm held horizontally across the front of the body).

I suggest that the skylab 3 results of Montgomery et al. [16] are of great significance. They support another hypothesis:

Hypothesis 2: Evolution in a $1 \mathrm{~g}$ environment has been

accompanied by the elaboration of a cellular machinery

for the maintenance of positional homeostasis and.

energetically, this is a costly investment.

If the data presented in Montgomery et al [16] are verified, then hypothesis 2 suggests that the energy for positional homeostasis inust be a major component of what is normally labeled basal metabolism. It further suggests that causes for the nutritional and physiological problems which have been encountered during space flight exposure must be sought at the level of cellular and tissue metabolic expenditure in the maintenance of positional homeostasis as well as at the level of exercise physiology. One is struck by several observations. First, the metabolism of calcium, so important to interceliular ligands, sustentacular tissues and contractile mechanisms, is perturbed in space in a direction suggesting reduced dependence on these entities which are of importance to positional homeostasis. Second, in metazoans the vital organs are situated in the body in such a manner as to minimize the frequency, rapidity and magnitude of exposure to changes in the direction of gravity. Thus, the vital organs of quadruped and biped animals are clustered about their longitudinal axis rather than being distributed in appendages. This minimizes the frequency and rapidity of changes in the orientation of these organs to the direction of gravity, and when such changes occur they seldom exceed an angel of $90^{\circ}$

While some aspects of these hypotheses can be tested on centrifuges at $g>1$, their full examination probably can not be accomplished on Earth. Carefully designed experiments in low-gravity environments will be needed. There is an additional need to explore the genetic parameters of the possible feedback control mechanisms discussed above relevant to the fabrication, maintenance and use of the structural and biochemical mechanisms demanded by the requirements for positional homeostasis.

These matters may also be related to questions concerning the initiation of development. It is significant that, during early development, mechanisms to maintain positional homeostasis have not yet becone established. Gravitational changes could be expected to have more pronounced effects at these stages than at later stages. This is particularly true for the egg which contains naked cytoplasm in mass aggregates and without any means of stabilization except through the agency of the cytoskeleton. It is to be expected that the instability of the cytoskeleton of the egg at the time of normal symmetrization would render this period particularly susceptible to gravitational changes $[4,19]$. 


\section{SUMMARY}

Every gram of mass in every organism on Earth is directly acted upon by gravity at a force of 980 dynes. Using relevant formulations and simplifying assumptions, it was shown that on Earth the force due to gravity imparts torque to cells, namely $2.5 \times 10^{-13}$ to $8.5 \times 10^{-1}$ dyne-cm for $6.4 \mathrm{\mu m}$ to $31 \mathrm{~mm}$ cells, respectively. These figures compare with $4.9 \times 10^{-9}$ dyne-cm calculated for 6 microtubules acting on a lever arm.

Since the presentation of cells to their environment is critical, it is evident that they maintain what is defined here as positional homeostasis. Energy to counteract the torque generated by gravity must be expended by the cell or its neighbors to maintain positional homeostasis, either dynamically or through the creation and maintenance of appropriate stabilizing structures.

At zero gravity this requirement for energy expenditure is zero. Thus celis in space may be expected to use less energy than do cells on Earth and this may or may not be accompanied by structural or biochemical changes depending upon the nature of mechanisms which maintain the machinery for positional homeostasis.

This hypothesis is supported by the skylab 3 results of Montgomery et al. [16] who present data suggesting that control cells on Earth used 58 more glucose than did the cells in space. This suggests that positional homeostasis is a costly component of basal metabolism.

The implications of this hypothesis for evolution, physiology, genetics and developmental biology both on Earth and in space are noted, including the conclusion that at the cellular and tissue level the postulation of special "gravity receptors" may not be necessary.

\section{ACKNOWLEDGEMENTS}

Supported by the Space Biology Program of the office of Space science and Applications, NASA Grant NAGW-29, and Ames Research Center Contract NAS 210945 .

Special appreciation is expressed to Karl Guthe of the University of Michigan Department of Zoology, and to William Parkinson of the University of Michigan Department of Physics for their helpful instruction and discussion, and to Richard Holmquist for playing the devil's advocate in his thoughtful editorial attention.

\section{IITERATURE CITED}

1. Gerhart, J., G. Ubbels, S. Black, K. Hara, and M. Kirschner. 1981. A reinvestigation of the role of the grey crescent in axis formation in Xenopus laevis. Nature 292:511-516.

2. Kirschner, M.W. and J.C. Gerhart. 1981. Spatial and temporal changes in the amphibian egg. Bioscience 31:381-388.

3. Malacinski, G.M. and Mae Moon Chung. 1981. Establishment of the site of involution at novel locations on the amphibian embryo. J. Morph. 169:149-159.

4. Nace, G.W. and J.W. Tremor. 1981. Clinostat exposure and symetrization of frog eggs. The Physiologist 24:577 - 578.

5. Pollard, E.C. 1971. Physical determinants of receptor mechanisms. In: S.A. Gordon and M.J. Cohen. Gravity and the Organism. University of Chicago Press.

6. Okada, T.S., K. Yasuda, H. Kondoh, K. Nomura, S. Takagi and K. Okuyama. 1982. Transdetermination and transdifferentiation of neural retinal cells into lens in culture. In: M.M. Burger and R. Weber. Embryonic Development, Part A: Genetic Aspects. Alan R, Liss, Inc., New York. pp. 249-255. 
7. Altman, P.I. and D.S. Dittmer, eds. 1962. Growth including Reproduction and Morphological Development. Federation of American Societies for Experimental Biology, Bethesda, Maryland. 608 pp.

8. Altman, P.I. and D.S. Dittner, eds. 1961. Blood and Other Body Fluids. Federation of American Societies for Experimental Biology, Washington, DC. 540 pp.

9. Weast, R.C., S.M. Selby and C.D. Hodgman, eds. 1964. Handbook of Chemistry and Physics. 45th Edition. The Chemical Rubber Company, Cleveland, Ohio.

10. Davidson, L. 1973. Contractile axopodia of the centrohelidan heliozoan, Heterophrys marina. J. Cell Biol. 59:71A.

11. Altman, P.L. and D.S. Dittmer, eds. 1966. Environmental Biology. Federation of American Societies for Experimental Biology, Bethesda, Maryland. $694 \mathrm{pp}$.

12. Masui, Y. 1982. Personal communication.

13. Opresko, L., H.S. Wiley and R.A. Wallace. 1980. Differential postendocytotic compartmentation in Xenopus oocytes is mediated by a specifically bound ligand. Cell 22:47-57.

14. Nieuwkoop, P.D. and J. Faber. 1956. Normal Table of Xenopus laevis (Daudin). A Systematical and Chronological Survey of the Development from the Fertilized Egg till the End of Metamorphosis. North-Holland Publishing Co., Amsterdam. 243 pp.

15. Romanoff, A.I. and A.J. Romanoff. 1949. The Avian Egg. John Wiley and Sons, Inc., New York.

16. Montgamery, P. O'B., Jr., J.E. Cox, R.C. Reynolds, J.S. Paul, L. Hayflick, D. Stock, W.W. Shulz, S. Kimzey, R.G. Thirolf, T. Rogers, D. Campbell and $J$. Murrell. 1977. The response of single human celis to zerogravity. In: R.S. Johnston and L.F. Dietlein. Biomedical Results From Skylab. N77-33780, Superintendent of Documents, U.S. Government Printing office, Washington, D.C. 20402. pp. 221-234.

17. Carlson, F.C. and D.R. Wilkie. 1974. Muscle Physiology. PrenticeHall, Englewood Cliffs, New Jersey.

18. Huxley, A.F. and R.M. Simmons. 1971, Proposed mechanism of force generation in striated muscle. Nature 233:533-538.

19. Tremor, J.W. and K.A. Souza. 1972. The influence of clinostat rotation on the fertilized amphibian egg. Space Iife Sciences $\underline{3}: 179-191$. 